\title{
Interfering with inflammation: a new strategy to block breast cancer self-renewal and progression?
}

\author{
Sara Cabodi and Daniela Taverna*
}

\begin{abstract}
Two recent studies show that epigenetics and inflammation play a relevant role in the regulation of transformation and cancer cell self-renewal in breast tumours, opening up the possibility that cancer progression can be controlled by interfering with inflammation cascades. Struhl's group showed that transient activation of the Src oncoprotein induces transformation and self-renewal of immortal cells via an epigenetic switch involving NF-KB, Lin28, Let-7 microRNA and IL-6. Concomitantly, Wicha's laboratory developed a strategy to selectively target cancer stem cells, retarding tumour growth and reducing metastasis by blocking the IL-8 receptor CXCR1 using either an inhibitor, repertaxin or a specific blocking antibody.
\end{abstract}

\section{Background}

Cancer development is a multistep process controlled by genetic perturbations such as activation of oncogenes or silencing of tumour suppressor genes and epigenetic events (that is, DNA methylation) occurring within a cell, as well as environmental influences (for instance, inflammation) [1-4]. The cancer stem cell (CSC) theory implies that tumours are generated and maintained by a small population of cells with both self-renewal and differentiation properties that contribute to tumorigenesis and cancer cell heterogeneity. CSCs are thought to be present in several haematological and solid tumours, including breast cancer [5], and to contribute to metastasis formation and tumour recurrence after therapy. In fact, CSCs seem to be involved in the acquirement of resistance to chemotherapy, radiotherapy or targeted agents [6-8], and could be the main reason for treatment failures. Consequently, a relevant effort is required for the identification of alternative treatments able to stop tumour progression and eradicate cancer.

*Correspondence: daniela.taverna@unito.it

Molecular Biotechnology Center, Dept. Oncological Sciences, University of Turin, Via Nizza, 52, 10126 Torino, Italy

\section{Articles}

Researchers from Struhl's group at Harvard University used the normal immortalized human mammary epithelial cell line MCF10A to evaluate the impact of a transient activation of the Src oncogene on transformation [9]. Interestingly, they observed that this single event was able to induce an epigenetic switch leading to a permanently transformed cell line, which could form self-renewing mammospheres containing CSCs. Src activation triggered an inflammatory response via IL-6 and created a permanent positive feedback loop involving NF- $\kappa$ B, Lin-28, Let-7 microRNA and STAT3.

In parallel, Ginestier and colleagues developed a strategy to target specifically breast CSCs by blocking the IL-8 receptor CXCR1 and therefore interfering with inflammation [10]. Indeed, using an inhibitor for CXCR1, repertaxin or a blocking antibody, they could deplete the CSC population of two breast cancer cell lines in vitro and could target the CSCs in xenografts in mice, retarding tumour growth and reducing metastasis formation. They further show that the effect of CXCR1 inhibition is mediated by the FAK/Akt pathway. Notably, cells presenting inactivated PTEN or overexpression of FAK are resistant to CXCR1 inhibition.

\section{Viewpoint}

The idea that a normal cell becomes transformed when several mutations accumulate permanently in its DNA is well accepted. The article by Iliopoulos and colleagues, however, reports for the first time that a transient event, such as activation of Src, is sufficient to mediate an epigenetic switch that leads to a stable transformed cell with self-renewing capacity - suggesting that not only mutations in DNA can contribute to cancer [9]. In addition, the fact that Src activation triggers an inflammatory response opens up the possibility that targeting an inflammatory signal at an earlier step (cytokine receptors) or at a later step (microRNA modulation) may be sufficient to reduce the CSC population. These are major discoveries in the field supported by solid experiments; owing to the important implications of these findings, however, it becomes essential to transfer Struhl's approach to a mouse model of tumorigenesis. 
Almost as a follow-up to Iliopoulos and colleagues' work, Ginestier and coworkers prove elegantly that targeting an inflammatory signal transduction pathway specific to CSCs makes reduction of tumour mass possible, overcoming the problem of chemoresistance [10]. The strength of this report is the fact that when using a cytotoxic agent alone, such as docetaxel, tumour mass is reduced but the CSC population is either not affected or is even increased. Conversely, repertaxin treatment alone or in combination with docetaxel significantly reduced the number of CSCs and led to the shrinkage of tumour mass by an indirect bystander effect mediated by FASL/FAS signalling.

Considering that repertaxin has already been used to reduce tissue damage after myocardial infarction or stroke [11] and that clinical phase I studies demonstrate a lack of toxicity for this compound, the use of this CXCR1 inhibitor in cancer therapy seems very promising. Consequently, it is possible that a synergistic negative effect on CSC growth or survival can be obtained by targeting various inflammatory signals at the same time, leading to a better outcome for several types of cancer.

In conclusion, these two articles present essential work for the breast cancer field and bring together three new concepts: tumorigenesis can be initiated by one or more transient epigenetic events; tumour progression can be achieved by the activation of inflammatory cascades; and tumour growth can be arrested or at least kept under control by blocking one or more CSC-specific inflammatory stimulus.

\section{Abbreviations}

CSC, cancer stem cell; IL, interleukin; NF, nuclear factor.

\section{Competing interests}

The authors declare that they have no competing interests.
Published: 28 April 2010

\section{References}

1. Hahn WC, Weinberg RA: Rules for making human tumor cells. N Engl J Med 2002, 347:1593-1603.

2. Baylin SB: DNA methylation and gene silencing in cancer. Nat Clin Pract Oncol 2005, 2(Suppl 1):S4-S11.

3. Bartel DP: MicroRNAs: target recognition and regulatory functions. Cell 2009, 136:215-233

4. Pierce BL, Ballard-Barbash R, Bernstein L, Baumgartner RN, Neuhouser ML, Wener MH, Baumgartner KB, Gilliland FD, Sorensen BE, McTiernan A, Ulrich CM: Elevated biomarkers of inflammation are associated with reduced survival among breast cancer patients. J Clin Oncol 2009, 27:3437-3444.

5. Visvader JE, Lindeman GJ: Cancer stem cells in solid tumours: accumulating evidence and unresolved questions. Nat Rev Cancer 2008, 8:755-768.

6. Phillips TM, McBride WH, Pajonk F: The response of CD24-/low/CD44+ breast cancer-initiating cells to radiation. J Nat/ Cancer Inst 2006, 98:1777-1785.

7. Yu F, Yao H, Zhu P, Zhang X, Pan Q, Gong C, Huang Y, Hu X, Su F, Lieberman J, Song E: Let-7 regulates self renewal and tumorigenicity of breast cancer cells. Cell 2007, 131:1109-1123.

8. Tanei T, Morimoto K, Shimazu K, Kim SJ, Tanji Y, Taguchi T, Tamaki Y, Noguchi S: Association of breast cancer stem cells identified by aldehyde dehydrogenase 1 expression with resistance to sequential paclitaxel and epirubicin-based chemotherapy for breast cancers. Clin Cancer Res 2009, 15:4234-4241.

9. Iliopoulos D, Hirsch HA, Struhl K: An epigenetic switch involving NF-KB, Lin28, Let-7 MicroRNA, and IL6 links inflammation to cell transformation. Cell 2009, 139:693-706.

10. Ginestier C, Liu S, Diebel ME, Korkaya H, Luo M, Brown M, Wicinski J, Cabaud O, Charafe-Jauffret E, Birnbaum D, Guan JL, Dontu G, Wicha MS: CXCR1 blockade selectively targets human breast cancer stem cells in vitro and in xenografts. J Clin Invest, 120:485-497.

11. Bertini R, Allegretti M, Bizzarri C, Moriconi A, Locati M, Zampella G, Cervellera MN, Di Cioccio V, Cesta MC, Galliera E, Martinez FO, Di Bitondo R, Troiani G, Sabbatini V, D'Anniballe G, Anacardio R, Cutrin JC, Cavalieri B, Mainiero F, Strippoli R, Villa P, Di Girolamo M, Martin F, Gentile M, Santoni A, Corda D, Poli G, Mantovani A, Ghezzi P, Colotta F: Noncompetitive allosteric inhibitors of the inflammatory chemokine receptors CXCR1 and CXCR2: prevention of reperfusion injury. Proc Natl Acad Sci U S A 2004, 101:11791-11796.

doi: $10.1186 /$ bcr 2563

Cite this article as: Cabodi A, Taverna D: Interfering with inflammation: a new strategy to block breast cancer self-renewal and progression? Breast Cancer Research 2010, 12:305. 